\title{
Forum Junge Radiologie der Deutschen Röntgengesellschaft (DRG) kooperiert mit Thieme
}

\author{
Kostenloser Zugriff auf die Wissensplattform eRef Radiologie mit allen relevanten \\ Weiterbildungsinhalten für den Facharzt
}

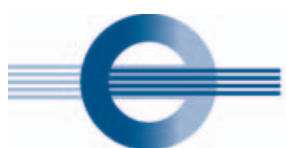

\author{
DEUTSCHE RÖNTGENGESELLSCHAFT \\ Gesellschaft für medizinische Radiologie e.V.
}

Stuttgart, März 2019 - Thieme hat gemeinsam mit dem Forum Junge Radiologie der Deutschen Röntgengesellschaft (DRG) ein digitales Modul entwickelt, das alle relevanten Informationen für das Weiterbildungscurriculum Radiologie bündelt. Dieses „Curriculum Radiologie“ ist pünktlich zur Eröffnung des 100. Röntgenkongresses am 29. Mai 2019 in der eRef Radiologie von Thieme abrufbar. Assistenzärztinnen und -ärzte, die als Neumitglieder in die DRG eintreten, erhalten eine 1-jährige kostenlose Mitgliedschaft in der DRG sowie einen 1-jährigen kostenlosen Zugang zur medizinischen Wissensplattform eRef Radiologie von Thieme. Damit können die Ärzte in Weiterbildung auf das Curriculum Radiologie und auf sämtliche radiologische Fachinformationen der Thieme-Gruppe zugreifen.

„Für junge Ärzte ist es nicht einfach, sich im Informationsdschungel zurecht zu finden. In der eRef Radiologie haben wir daher jetzt unsere Leseempfehlungen und Links im sogenannten „Curriculum Radiologie“ zusammengestellt. Hier finden junge Ärzte alle Fachinformationen, die sie sich für den Facharzt Radiologie aneignen müssen“, sagt Dr. Thekla H. Oechtering, Vorstandsvorsitzende des Forums Junge Radiologie. „Das Wissensportal eRef bietet hierfür den idealen Rahmen." Aufwendiges Suchen entfällt und es bleibt mehr Zeit zum Lesen, Lernen und für den Klinikalltag. Neben Auszügen aus radiologischen Büchern und Zeitschriften sowie Referenzabbildungen der Thieme-Gruppe finden sich im Curriculum Radiologie auch Links auf weiteres Lernmaterial, wie beispielsweise Inhalte der DRG und Leitlinien.
Thieme und die DRG gewähren den jungen Ärzten in Weiterbildung, die neu in die DRG eintreten, den kostenlosen Zugang zur eRef Radiologie für 1 Jahr. „Die kostenlose Mitgliedschaft in der DRG im ersten Jahr sowie die kostenlose Nutzung der eRef Radiologie ist ein guter Anreiz, Mitglied im Forum Junge Radiologie der DRG zu werden und sich auch berufspolitisch zu engagieren “, so Oechtering.

Die eRef bietet für alle medizinischen Fachgebiete den digitalen Zugriff auf die Inhalte der medizinischen Fachbücher, Zeitschriften und Datenbanken von Thieme und ausgewählten weiteren Fachverlagen, ein umfangreiches Bildarchiv, Videos und weitere Services. Damit können Ärzte in den verschiedenen Arbeitssituationen die jeweils relevanten Informationen schnell abrufen. Die eRef steht Kliniken, niedergelassenen Ärzten und Einrichtungen im Gesundheitswesen nach Bedarf vollumfänglich oder auf bestimmte Fachgebiete fokussiert zur Verfügung. Mitarbeiter, die in einer Klinik arbeiten, die die eRef lizenziert hat, können sich ihren persönlichen Account anlegen und haben damit jederzeit von jedem Endgerät aus Zugang und können die eRef-App nutzen.

Hier geht es zur eRef: https://eref. thieme.de/

\section{Die Thieme-Gruppe}

Thieme ist marktführender Anbieter von Informationen und Services, die dazu beitragen, Gesundheit und Gesundheitsversorgung zu verbessern. Das Familienunternehmen entwickelt mit seinen über 1000 Mitarbeiterinnen und Mitarbeitern digitale und analoge Angebote in Medizin und Chemie. Die internationale Unternehmensgruppe mit weltweit 11 Standorten nutzt dafür ein breites Experten- und Partnernetzwerk sowie die qualitativ hochwertigen Inhalte aus 200 Fachzeitschriften und 4400 Buchtiteln. Mit ihren Lösungsangeboten unterstützt Thieme relevante Informationsprozesse in der Wissenschaft, in Ausbildung und Patientenversorgung. Medizinstudierende, Ärzte, Pflegekräfte und Therapeuten, Kliniken, Krankenkassen sowie alle an Gesundheit Interessierten stehen hierbei im Mittelpunkt. Anspruch der Thieme-Gruppe ist es, ihnen genau die Informationen, Services und Werkzeuge bereitzustellen, die sie in einer bestimmten Arbeitssituation oder Lebensphase benötigen. Durch die hohe Qualität und zielgruppenspezifische Relevanz der angebotenen Leistungen bereitet Thieme den Weg für eine bessere Medizin und mehr Gesundheit im Leben.

\section{Die Deutsche Röntgengesell- schaft}

Die Deutsche Röntgengesellschaft e. V. (DRG) ist eine medizinische Fachgesellschaft, die die Radiologie in ihren medizinischen Anwendungen, ihrer qualifizierenden Vermittlung und in ihrer wissenschaftlichen Weiterentwicklung unterstützt und befördert. Sie leistet damit einen aktiven Beitrag für eine qualitätsgesicherte, wissenschaftlich fundierte Anwendung aller bildgebenden Verfahren und minimalinvasiven Therapien unter besonderer Berücksichtigung des Strahlenschutzes. Ziel der DRG ist es, die Radiologie als einen festen, unabdingbaren Teil fachärztlicher Leistung in Deutschland nachhaltig zu verankern und als Impulsgeber für Innovationen im medizinischen Versorgungssystem zu etablieren. Sie wird dabei von der Überzeugung geleitet, dass die Radiologie mit ihren bild- 
gebenden Verfahren und minimalinvasiven Therapien integraler Bestandteil und wesentliche Voraussetzung für eine qualitativ hochwertige, wirtschaftliche und stets am Patienten ausgerichtete medizinische Grundversorgung in Deutschland ist.

\section{Pressekontakt}

Carola Schindler

Thieme Kommunikation

Georg Thieme Verlag KG

ein Unternehmen der Thieme-Gruppe

Rüdigerstraße 14, 70469 Stuttgart

Fon + $497118931-488$

Fax $+497118931-167$

carola.schindler@thieme.de
Dr. Hans-Georg Stavginski

Bereichsleitung Kommunikation

Deutsche Röntgengesellschaft e. V. Ernst-Reuter-Platz 10, 10587 Berlin

Fon + $4930916070-43$

Fax + $4930916070-22$

stavginski@drg.de

Bibliografie

DOI https://doi.org/10.1055/a-0864-6454 Online-Publikation: 2019

Fortschr Röntgenstr 2019; 191: 287-288

(c) Georg Thieme Verlag KG, Stuttgart · New York ISSN 1438-9029 